FT/UCM-80-2001

\title{
Computing noncommutative Chern-Simons theory radiative corrections on the back of an envelope
}

\author{
C. P. Martín† \\ Departamento de Física Teórica I, Universidad Complutense, 28040 Madrid, Spain
}

In memory of George Leibbrandt

\begin{abstract}
We show that the renormalized $U(N)$ noncommutative Chern-Simons theory can be defined in perturbation theory so that there are no loop corrections to the 1PI functional of the theory in an arbitrary homogeneous axial (time-like, light-like or space-like) gauge. We define the free propagators of the fields of the theory by using the Leibbrandt-Mandelstam prescription - which allows Wick rotation and is consistent with power-counting- and regularize its Green functions with the help of a family of regulators which explicitly preserve the infinitesimal vector Grassmann symmetry of the theory. We also show that in perturbation theory the nonvanishing Green functions of the elementary fields of the theory are products of the free propagators.
\end{abstract}

\section{1.- Introduction}

In 1982 [1], the integral of the Chern-Simons three-form was added to the Yang-Mills action to give the gauge field in three dimensions a mass without the need of the Higgs mechanism. Thus Topologically Massive Yang-Mills theory was born. In 1988, Nonabelian Chern-Simons theory [2] -the theory whose action is the integral over a given (commutative) three-dimensional manifold of the Chern-Simons three form for a nonabelian gauge group- was introduced to give a three-dimensional and field theoretical definition of the Jones polynomial and other topological invariants of three-dimensional manifolds. When the gauge field of the Chern-Simons action couples to matter fields we obtain field theories having anyonic excitations, these field theories being relevant in the long wavelength range description of the fractional quantum Hall effect and high temperature conductivity [3].

The perturbative construction of Nonabelian Chern-Simons theory has been the subject of intensive research and has yielded very beautiful and deep results -see ref. [4] and references therein. In particular, the perturbative study of the observables of the theory in the temporal and light-cone gauges has given rise [5] respectively to a combinatorial representation of the Vassiliev invariants and the representation of these invariants furnished by the Kontsevich integral. The Light-cone gauge had been used much earlier [6] to quantize Nonabelian Chen-Simons theory and obtain the Knizhnik-Zamolodchikov equation as the differential equation to be satisfied by the Wilson line vevs, thus making connection with the Wess-Zumino-Witten model.

$\dagger$ E-mail: carmelo@elbereth.fis.ucm.es 
The Chern-Simons action over noncommutative manifolds [7] -henceforth the noncommutative Chern-Simons action- was first considered in refs. [8]. The properties of Chern-Simons theory on the noncommutative plane -henceforth noncommutative ChernSimons theory- has been studied perturbatively [9] and nonperturbatively [10, 11] in a number of papers. The noncommutative Chern-Simons action as an induced action has been considered in ref. [12]. Fractional quantum Hall fluids can be given an effective description by using the noncommutative Chern-Simons theory [13]. It would appear that noncommutative Chern-Simons theory will play in Physics as outstanding a role as its ordinary sibling does.

The purpose of this paper is to quantize perturbatively the noncommutative ChernSimons theory in a general homogeneous axial-type gauge $-n^{\mu} \mathrm{A}_{\mu}=0$, either with $n^{2}>0$ (space-like), $n^{2}=0$ (light-cone), or with $n^{2}<0$ (time-like). We shall use the generalized Leibbrandt-Mandelstam prescription to go around the axial infrared singularity of the naive propagators and thus treat on equal footing the three types of axial gauges. By employing a rather arbitrary regularization method which explicitly preserves the Grassmann vector symmetry of the theory, we shall be able to compute, at any order in perturbation theory, the radiative corrections to Green functions of the elementary fields. We shall thus show that in the theory so defined these Green functions lack radiative corrections and that they are, the Green functions, either products of the free propagators or vanish.

The layout of this paper is as follows. In section 2, we establish the symmetries of the gauge fixed theory and perform the computation of the quantum corrections to the 1PI functional. Section 3 contains the conclusions and some comments.

\section{2.- Symmetries and quantum corrections}

To define in perturbation theory the noncommutative $U(N)$ Chern-Simons theory in an homogeneous axial type gauge one defines, perturbatively, the path integral with the help of the following action

$S=-\frac{k}{4 \pi} \operatorname{Tr} \int d^{3} x \epsilon^{\mu \rho \nu}\left(\mathrm{A}_{\mu} \star \partial_{\rho} \mathrm{A}_{\nu}-\frac{2 i}{3} \mathrm{~A}_{\mu} \star \mathrm{A}_{\rho} \star \mathrm{A}_{\nu}\right)+2 \operatorname{Tr} \int d^{3} x\left(\mathrm{~b} \star n^{\mu} \mathrm{A}_{\mu}-\overline{\mathrm{c}} \star n^{\mu} \mathrm{D}_{\mu}[\mathrm{A}] \mathrm{c}\right)$,

where $\mathrm{D}_{\mu}[\mathrm{A}] \mathrm{c}=\partial_{\mu} \mathrm{c}-i\left(\mathrm{~A}_{\mu} \star \mathrm{c}-\mathrm{c} \star \mathrm{A}_{\mu}\right)$. The fields $\mathrm{A}_{\mu}, \mathrm{b}, \overline{\mathrm{c}}$ and $\mathrm{c}$ take values in the Lie algebra of $U(N)$. We define the generators of this Lie algebra as $N \times N$ hermitian matrices, $\left\{\left(T^{a}\right)_{j}^{i}\right\}_{a=0, \ldots, N^{2}-1}, i, j=1, . ., N$, which satisfy $\left[T^{a}, T^{b}\right]=-i f^{a b c} T^{c}$ and which are normalized so that $\operatorname{Tr} T^{a} T^{b}=\frac{1}{2} \delta^{a b}$, if $a, b \geq 1$, and $T^{0}=\frac{1}{\sqrt{2 N}}$. For this normalization of the generators, $T^{a}$, the path integral is invariant under arbitrary gauge transformations only if $k$ is an integer [11] -a result which also holds for the noncommutative torus [8].

The action in eq. (1) is invariant under BRS, $s$, and antiBRS, $\bar{s}$, transformations:

$$
\begin{aligned}
& s \mathrm{~A}_{\mu}(x)=D_{\mu} c(x), s \bar{c}(x)=\mathrm{b}, s \mathrm{~b}(x)=0, s \mathrm{c}(x)=i(\mathrm{c} \star \mathrm{c})(x) \\
& \bar{s} \mathrm{~A}_{\mu}(x)=D_{\mu} \overline{\mathrm{c}}(x), \bar{s} \mathrm{c}(x)=b, \bar{s} \mathrm{~b}(x)=0, s \overline{\mathrm{c}}(x)=i(\overline{\mathrm{c}} \star \overline{\mathrm{c}})(x) .
\end{aligned}
$$

As in the commutative space-time case, the action in eq. (1) is also invariant under the following infinitesimal transformations

$$
v_{\mu} \mathrm{A}_{\nu}(x)=\frac{4 \pi}{k} \epsilon_{\mu \nu \rho} n^{\rho} \overline{\mathrm{c}}(x), v_{\mu} \overline{\mathrm{c}}(x)=0, v_{\mu} \mathrm{b}(x)=\partial_{\mu} \overline{\mathrm{c}}(x), v_{\mu} \mathrm{c}(x)=\mathrm{A}_{\mu}(x)
$$




$$
\bar{v}_{\mu} \mathrm{A}_{\nu}(x)=\frac{4 \pi}{k} \epsilon_{\mu \nu \rho} n^{\rho} \mathrm{c}(x), \bar{v}_{\mu} \mathrm{c}(x)=0, \bar{v}_{\mu} \mathrm{b}(x)=\partial_{\mu} \mathrm{c}(x), \bar{v}_{\mu} \overline{\mathrm{c}}(x)=\mathrm{A}_{\mu}(x) .
$$

The BRS transformations, the transformations generated by $v_{\mu}$ and the operator $\partial_{\mu}$ close upon imposing the equations of motion; thus generating the following trivial noncommutative generalization of the on-shell $N=1$ supersymmetry algebra over commutative space-time of ref. [14]:

$$
s^{2}=0,\left\{v_{\mu}, v_{\nu}\right\}=0,\left\{s, v_{\mu}\right\}=\partial_{\mu}
$$

The infinitesimal transformations generated by $\bar{v}_{\mu}$ were introduced in ref. [15], but they do not lead to an on-shell closed algebra.

To make sense out of the Feynman diagrams which yield the 1PI functional of the theory with action in eq. (1), we need to find first our way around the axial IR singularity, $p n=0$, of the free propagators, i.e., we need to define the object

$$
\frac{1}{p n}
$$

as a distribution. This we shall do by using the generalized Leibbrandt-Mandelstam prescription:

$$
\frac{1}{p n} \equiv \frac{p n^{*}}{p n p n^{*}+i 0^{+}},
$$

where $n^{*}$ is a suitable vector. If space-time is commutative, the generalized LeibbrandtMandelstam prescription allows, unlike the principal-value prescription, Wick rotation of the Green functions whatever the axial-type gauge chosen -the light-cone gauge, in particular- and whatever the space-time dimension. The prescription in eq. (3) can thus be used to define Green functions whose UV finiteness may be settled by using powercounting arguments [16]. This property also holds for theories defined on noncommutative Minkowski space-times of electric type -and light-like type- since the Moyal phases of the interaction vertices do not preclude Wick rotation to Euclidean noncommutative spacetime [17]. In this paper we shall not need to use any particular particular realization of $n^{*}$, all we shall use are the aforementioned properties of the Leibbrandt-Mandelstam prescription. The reader is referred to refs. [18], [19] and [20] for further information on the generalized Leibbrandt-Mandelstam prescription.

Now that we have defined the free propagators, we may go on and check whether loop corrections to the Green functions are UV finite or they give rise to UV divergences. The same type of power-counting arguments as in the ordinary case $[16,15]$ can be applied to the planar contribution of the Green functions to conclude that the noncommutative theory presents UV divergences. To regulate these divergences we shall use the family of regulators introduced in ref. [15]. These regularizations render absolutely convergent the Feynman diagrams of the theory upon Wick rotation to Euclidean noncommutative space-time. Furthermore, they preserve explicitly the infinitesimal symmetries in eq. (2), for the regularized action reads:

$$
\begin{aligned}
S_{\Lambda}= & -\frac{k}{4 \pi} \operatorname{Tr} \int d^{3} x \epsilon^{\mu \rho \nu}\left[\mathrm{A}_{\mu} \star \mathrm{R}\left(-\frac{\partial^{2}}{\Lambda^{2}}\right) \partial_{\rho} \mathrm{A}_{\nu}-\frac{2 i}{3} \mathrm{~A}_{\mu} \star \mathrm{A}_{\rho} \star \mathrm{A}_{\nu}\right] \\
& +2 \operatorname{Tr} \int d^{3} x\left[\mathrm{~b} \star \mathrm{R}\left(-\frac{\partial^{2}}{\Lambda^{2}}\right) n^{\mu} \mathrm{A}_{\mu}-\overline{\mathrm{c}} \star \mathrm{R}\left(-\frac{\partial^{2}}{\Lambda^{2}}\right) n^{\mu} \partial_{\mu} \mathrm{c}+i \overline{\mathrm{c}} \star\left(\mathrm{A}_{\mu} \star \mathrm{c}-\mathrm{c} \star \mathrm{A}_{\mu}\right)\right] .
\end{aligned}
$$


$\mathrm{R}\left(-\frac{\partial^{2}}{\Lambda^{2}}\right)$ is any of the functions below:

$$
\mathrm{R}\left(-\frac{\partial^{2}}{\Lambda^{2}}\right)=e^{-\frac{\partial^{2}}{\Lambda^{2}}}, \quad \mathrm{R}\left(-\frac{\partial^{2}}{\Lambda^{2}}\right)=\left(1+\frac{\partial^{2}}{\Lambda^{2}}\right)^{m}, m \geq 2, m \text { an integer. }
$$

The regularized action in eq. (4) is not BRS invariant - neither antiBRS invariant. However, we shall show that

$$
\Gamma_{\Lambda}=S_{\Lambda}
$$

$\Gamma_{\Lambda}$ being the $1 \mathrm{PI}$ functional for the action in eq. (4), so that the limit

$$
\lim _{\Lambda \rightarrow \infty} \Gamma_{\Lambda}=S
$$

$S$ being the action in eq. (1), yields a renormalized theory with no loop corrections to the tree-level 1PI functional. Obviously, the renormalized theory thus defined is invariant under BRS and antiBRS transformations, let alone the transformations in eq. (2). Notice that for the regularizations chosen, which explicitly preserve the infinitesimal vector symmetries in eq. (2), no shift of the Chern-Simons parameter $k$ occurs. The regularization method used in ref. [21] will yield, presumably, such a shift, at the cost of spoiling the supersymmetry invariance of the theory defined by eq. (6)-see ref. [22] for the "commutative" analysis.

The Feynman rules for the action in eq. (4) are given in Fig. 1, where

$$
\mathrm{V}_{j_{1} j_{2} j_{3}}^{i_{1} i_{2} i_{3}}\left(k_{1}, k_{2}\right)=\delta_{j_{3}}^{i_{1}} \delta_{j_{1}}^{i_{2}} \delta_{j_{2}}^{i_{3}} e^{\frac{i}{2} \theta\left(k_{1}, k_{2}\right)}-\delta_{j_{2}}^{i_{1}} \delta_{j_{3}}^{i_{2}} \delta_{j_{1}}^{i_{3}} e^{-\frac{i}{2} \theta\left(k_{1}, k_{2}\right)}
$$

Let us move on and show that eq. (5) holds indeed. Let us call a vertex of a 1PI Feynman diagram internal if none of its legs is an external leg. Every 1PI Feynman diagram with two loops or more contains at least one internal vertex. An internal vertex can be of A-A-A-type or of $\overline{\mathrm{c}}-\mathrm{A}$-c-type. The contribution to the diagram coming from each of these vertices with their legs on reads

$$
\begin{aligned}
& -\mathrm{V}_{i_{1} i_{2} i_{3}}^{j_{1} j_{2} j_{3}}\left(k_{1}, k_{2}\right) \prod_{i=1}^{3} \frac{1}{R\left(k_{i}^{2} / \Lambda^{2}\right)} \frac{k_{i} n^{*}}{k_{i} n k_{i} n^{*}+i 0^{+}} \epsilon^{\nu_{1} \nu_{2} \nu_{3}} \epsilon_{\mu_{1} \rho_{1} \nu_{1}} \epsilon_{\mu_{2} \rho_{2} \nu_{2}} \epsilon_{\mu_{3} \rho_{3} \nu_{3}} n^{\rho_{1}} n^{\rho_{2}} n^{\rho_{3}}, \\
& -\frac{\pi}{2 k} \mathrm{~V}_{i_{1} i_{2} i}^{j_{1} j_{2} j}\left(k_{1}, k_{2}\right) \prod_{i=1}^{3} \frac{1}{R\left(k_{i}^{2} / \Lambda^{2}\right)} \frac{k_{i} n^{*}}{k_{i} n k_{i} n^{*}+i 0^{+}} \epsilon_{\mu \rho \sigma} n^{\rho} n^{\sigma},
\end{aligned}
$$

respectively. The second contribution obviously vanishes and so it does the first one since $\epsilon^{\nu_{1} \nu_{2} \nu_{3}} \epsilon_{\mu_{2} \rho_{2} \nu_{2}} \epsilon_{\mu_{3} \rho_{3} \nu_{3}} n^{\rho_{2}} n^{\rho_{3}}=n^{\nu_{1}} \epsilon_{\mu_{2} \mu_{3} \sigma} n^{\sigma}$. We have thus shown that beyond one-loop there are no contributions to the 1PI functional of the regularized theory defined by the action in eq. (4). Now for the one-loop loop contributions.

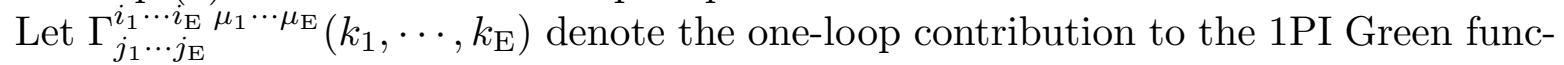
tion of $\mathrm{E}$ gauge fields and no ghost field. If $\mathrm{E} \geq 3$, then

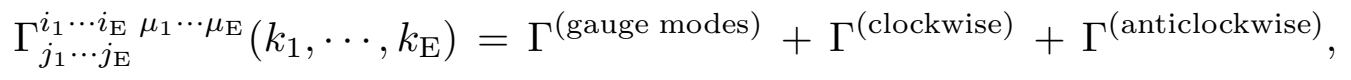



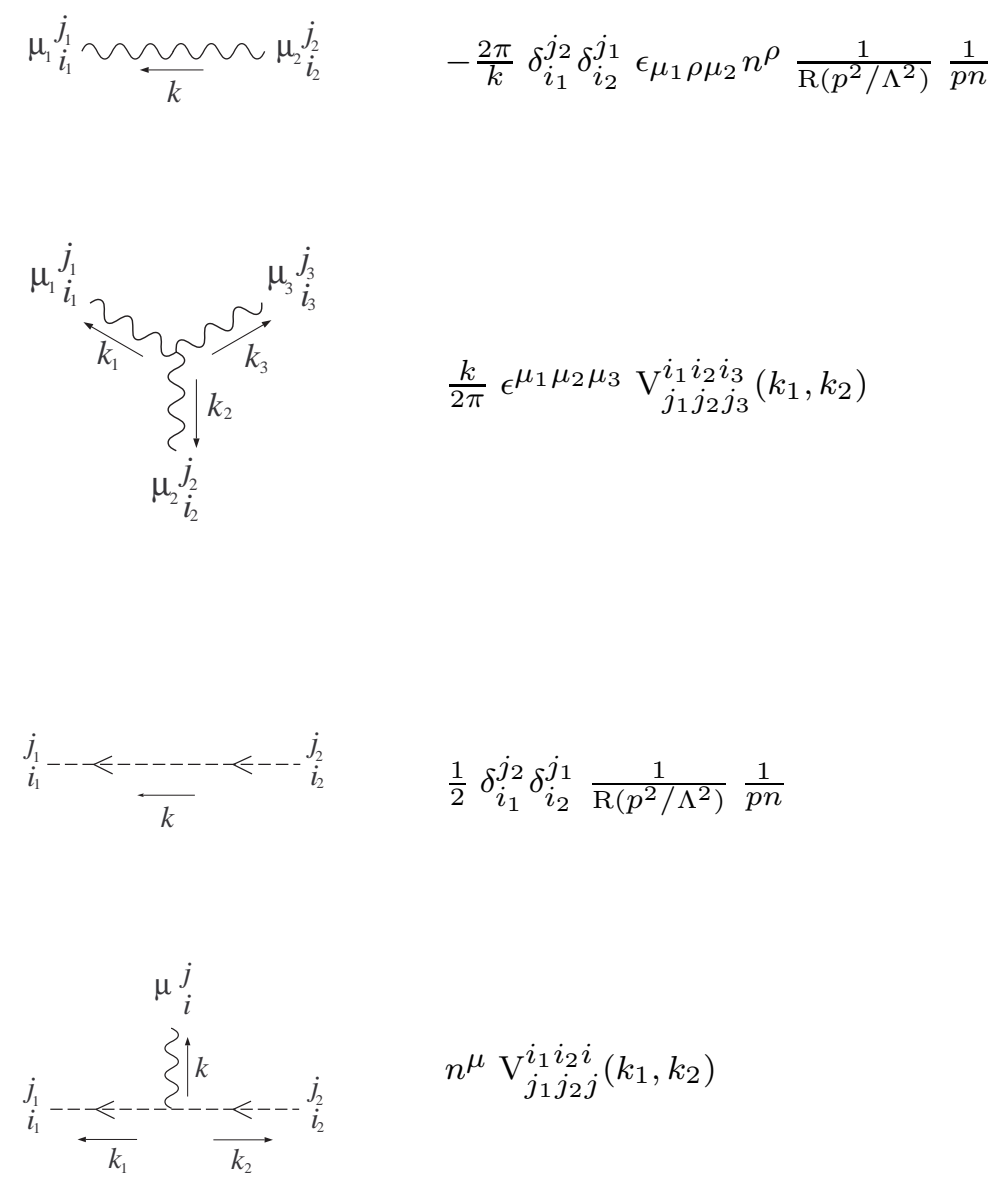

Figure 1.- Feynman rules for noncommutative $U(N)$ Chern-Simons theory in a homogeneous axial gauge. Note that $\mathrm{V}_{j_{1} j_{2} j_{3}}^{i_{1} i_{2} i_{3}}\left(k_{1}, k_{2}\right)=\delta_{j_{3}}^{i_{1}} \delta_{j_{1}}^{i_{2}} \delta_{j_{2}}^{i_{3}} e^{\frac{i}{2} \theta\left(k_{1}, k_{2}\right)}-\delta_{j_{2}}^{i_{1}} \delta_{j_{3}}^{i_{2}} \delta_{j_{1}}^{i_{3}} e^{-\frac{i}{2} \theta\left(k_{1}, k_{2}\right)}$.

where $\Gamma^{\text {(gauge modes) }}, \Gamma^{\text {(clockwise) }}$ and $\Gamma^{\text {(anticlockwise) }}$ are, respectively, the contributions furnished by gauge modes going around the loop and ghost modes propagating around the loop clockwise and anticlockwise. Using the Feynman rules in fig. 1, one can readily show that

$$
\begin{aligned}
& \Gamma^{\text {(gauge modes })}=\int \frac{d^{3} q}{(2 \pi)^{3}} \prod_{i=1}^{\mathrm{E}} \frac{1}{R\left(p_{i}^{2} / \Lambda^{2}\right)} \frac{p_{i} n^{*}}{p_{i} n p_{i} n^{*}+i 0^{+}} \prod_{i=1}^{\mathrm{E}} \mathrm{V}_{j_{i} l_{i-1} m_{i}}^{i_{i} m_{i-1} l_{i}}\left(-k_{i}, p_{i-1}\right) \prod_{i=1}^{\mathrm{E}} \mathrm{N}_{\nu_{i}}^{\mu_{i} \nu_{i-1}} \\
& \Gamma^{(\text {clockwise })}=-(-1)^{\mathrm{E}} \prod_{i=1}^{\mathrm{E}} n^{\mu_{i}} \int \frac{d^{3} q}{(2 \pi)^{3}} \prod_{i=1}^{\mathrm{E}} \frac{1}{R\left(p_{i}^{2} / \Lambda^{2}\right)} \frac{p_{i} n^{*}}{p_{i} n p_{i} n^{*}+i 0^{+}} \prod_{i=1}^{\mathrm{E}} \mathrm{V}_{j_{i} l_{i-1} m_{i}}^{i_{i} m_{i-1} l_{i}}\left(-p_{i-1}, p_{i}\right)
\end{aligned}
$$

and

$$
\Gamma^{(\text {anticlockwise })}=-(-1)^{\mathrm{E}} \prod_{i=1}^{\mathrm{E}} n^{\mu_{i}} \int \frac{d^{3} q}{(2 \pi)^{3}} \prod_{i=1}^{\mathrm{E}} \frac{1}{R\left(p_{i}^{2} / \Lambda^{2}\right)} \frac{p_{i} n^{*}}{p_{i} n p_{i} n^{*}+i 0^{+}} \prod_{i=1}^{\mathrm{E}} \mathrm{V}_{l_{i-1} m_{i-1} m_{i} i_{i}}^{m_{i}}\left(-p_{i-1}, p_{i}\right) .
$$

In the previous equation $l_{0}, m_{0}, v_{0}$ and $p_{0}$ are equal to $l_{\mathrm{E}}, m_{\mathrm{E}}, v_{\mathrm{E}}$ and $p_{\mathrm{E}}$, respectively, 
and $p_{1}=q$ and $k_{i}-p_{i-1}+p_{i}=0 . k_{i},-p_{i-1}$ and $p_{i}$ are the momenta coming out of the vertex with index $i$. Further, the symbol $\mathrm{N}_{\nu_{i}}^{\mu_{i} \nu_{i-1}}$ denotes the tensor contraction $-\epsilon^{\mu_{i} \nu_{i-1} \rho_{i}} \epsilon_{\rho_{i} \sigma \nu_{i}} n^{\sigma}$.

Now, it is not difficult to show that

$$
\prod_{i=1}^{\mathrm{E}} \mathrm{N}_{\nu_{i}}^{\mu_{i} \nu_{i-1}}=2(-1)^{\mathrm{E}} \prod_{i=1}^{\mathrm{E}} n^{\mu_{i}}, \quad \mathrm{~V}_{m_{i} l_{i-1} j_{i}}^{l_{i} m_{i-1} i_{i}}\left(p_{i},-p_{i-1}\right)=-\mathrm{V}_{j_{i} l_{i-1} m_{i}}^{i_{i} m_{i-1} l_{i}}\left(-p_{i-1}, p_{i}\right)
$$

and

$$
\mathrm{V}_{j_{i} l_{i-1} m_{i}}^{i_{i} m_{i-1} l_{i}}\left(-p_{i-1}, p_{i}\right)=\mathrm{V}_{j_{i} l_{i-1} m_{i}}^{i_{i} m_{i-1} l_{i}}\left(-k_{i}, p_{i-1}\right), \mathrm{V}_{l_{i-1} m_{i} j_{i}}^{m_{i-1} l_{i} i_{i}}\left(-p_{i-1}, p_{i}\right)=\mathrm{V}_{j_{i} l_{i-1} m_{i}}^{i_{i} m_{i-1} l_{i}}\left(-k_{i}, p_{i-1}\right)
$$

which employed along with eq. (8) yield

$$
\Gamma^{(\text {clockwise })}=\Gamma^{(\text {anticlockwise })}=-\frac{1}{2} \Gamma^{\text {(gauge modes })}
$$

If we substitute this result in eq. (7), we will conclude that

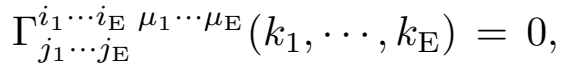

whenever $\mathrm{E} \geq 2$. This result also holds for $\mathrm{E}=1,2$ since in both these cases

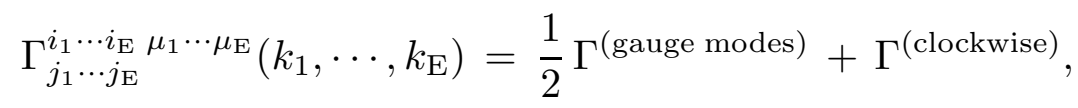

with $\Gamma^{\text {(gauge modes) }}$ and $\Gamma^{\text {(clockwise) }}$ given in eq. (8). Notice that the factor $\frac{1}{2}$ on the r.h.s of eq. (8) is the symmetry factor of the diagram with gauge modes propagating around the loop.

The one-loop contribution to any 1PI Feynman diagram with ghost vanishes since it has at least an internal gauge propagator contracted with the $n^{\mu}$ vector of a $\bar{c}$-A-c vertex, a contraction which vanishes. 


\section{3.- Conclusions and comments}

We have shown in this paper that $U(N)$ Chern-Simons theory on the noncommutative plane can be defined in perturbation theory so that its 1PI functional in an arbitrary homogeneous axial gauge receives no quantum corrections. To construct the theory we have used, as an intermediate stage, a regularization method which preserves the infinitesimal vector Grassmann symmetry of the theory.

Let us state next that for our definition of the theory the following results hold

$$
\begin{aligned}
& \left\langle\prod_{l=1}^{2 \mathrm{E}+1} \mathrm{~A}_{j_{l} \mu_{l}}^{i_{l}}\left(x_{l}\right)\right\rangle=0, \\
& \left\langle\prod_{l=1}^{2 \mathrm{E}} \mathrm{A}_{j_{l} \mu_{l}}^{i_{l}}\left(x_{l}\right)\right\rangle=\sum_{\mathrm{P}} \prod_{m=1}^{\mathrm{E}}\left\langle\mathrm{A}_{j_{l_{m}} \mu_{l_{m}}}^{i_{l_{m}}}\left(x_{l_{m}}\right) \mathrm{A}_{j_{n_{m}} \mu_{n_{m}}}^{i_{n_{m}}}\left(x_{n_{m}}\right)\right\rangle,
\end{aligned}
$$

where $\mathrm{P}=\left\{\left(l_{1}, n_{1}\right),\left(l_{2}, n_{2}\right), \cdots,\left(l_{\mathrm{E}}, n_{\mathrm{E}}\right)\right\}$ is the set of all possible collections of $\mathrm{E}$ pairings of the elements of $\{1, \cdots E\}$ with $l_{m}<n_{m}$. To prove the results in eq. (9), one may use the invariance of the theory under the first infinitesimal transformation in eq. (2), the fact that by ghost number conservation

$$
\left\langle\prod_{l=1}^{\mathrm{E}} \mathrm{A}_{j_{l} \mu_{l}}^{i_{l}}\left(x_{l}\right) \mathrm{c}_{j_{\mathrm{E}+1}}^{i_{\mathrm{E}+1}}\left(x_{\mathrm{E}+1}\right)\right\rangle=0,
$$

and the fact that for our definition of the noncommutative theory

$$
\left\langle\mathrm{O}_{1}(\mathrm{~A}) \mathrm{O}_{2}(\mathrm{c} \overline{\mathrm{c}})\right\rangle=\left\langle\mathrm{O}_{1}(\mathrm{~A})\right\rangle\left\langle\mathrm{O}_{2}(\mathrm{c} \overline{\mathrm{c}})\right\rangle .
$$

Hence, the Green functions of the elementary fields of the noncommutative theory are in perturbation theory those of the ordinary (over commutative space-time) theory. Of course, as happens in the ordinary case, it is the vev of gauge invariant operators which carries the really nontrivial information -barring the famous one-loop shift of $k$. We will report elsewhere on the equations satisfied by vev of Wilson lines and the connection of noncommutative Chern-Simons theory with the noncommutative Wess-Zumino-Witten model [23].

Finally, when I was writing this paper, I came across the paper quoted in ref. [24] where radiative corrections in Noncommutative Chern-Simons theory in an axial spacelike gauge are analyzed. In the latter paper the principal value prescription is used and the results therein displayed agree with ours for space-like gauges.

\section{Acknowledgments}

This work has been partially supported by CICyT under grant PB98-0842. As I was writing the closing sentences of this paper I was informed of the demise of Professor George Leibbrandt; to him I dedicate this paper with all my everlasting gratitude. Requiescat In Pace. 


\section{References}

[1] S. Deser, R. Jackiw and S. Templeton, Ann. Phys. 140 (1982) 372.

[2] E. Witten, Comm. Math. Phys. 121 (1989) 351.

[3] R. Mackenzie and F. Wilczek, Int. J. Mod. Phys. A3 (1988) 2827; Y.H. Chen, F. Wilczek, E. Witten and B. I. Halperin, Int. J. Phys. B3 (1989) 1001; A. Lopez and E. Fradkin, "Fermionic Chern-Simons Theory for the Fractional Hall Effect" in Composite Fermions in the Quantum Hall Effect, edited by O, Heinonen, condmat/9704055; S.H. Simon, "The Chern-Simons Fermi Liquid Description of the Quantum Hall States" in Composite Fermions", ed. O. Heinonen, World Scientific, condmat/9812186; G.V. Dunne, "Aspects of Chern-Simons Theory", lectures in Topological Aspects of Low Dimensional Systems, Les Houches Summer School 1998, hepth/9902115.

[4] J.M.F. Labastida, "Chern-Simons Gauge Theory: Ten Years After", lecture delivered at the workshop "Trends in Theoretical Physics II", Buenos Aires, November 1998, hep-th/9905057.

[5] J.M.F. Labastida and Esther Perez, J.Math.Phys. 39 (1998) 5183-5198; J.Math.Phys. 41 (2000) 2658-2699.

[6] S. King and J. Frölich, Comm. Math. Phys. 126 (1989) 167.

[7] J.M. Gracia-Bondía, J.C. Várilly and H. Figueroa, "Elements of Noncommutative Geometry", Birkäuser, 2001.

[8] A. Chamseddine and J. Frölich, J. Math. Phys. 35 (1994) 5195; T. Krajewski, "Gauge invariance of the Chern-Simons action in noncommutative geometry", math-ph/990 3047.

[9] A. A. Bichl, J. M. Grimstrup, V. Putz and M. Schweda, JHEP 0007 (2000) 046; Guang-Hong Chen and Yong-Shi Wu, Nucl.Phys. B593 (2001) 562.

[10] A. P. Polychronakos, JHEP 0011 (2000) 008; J. Kluson, "Matrix model and ChernSimons theory", hep-th/0012184; M.M. Sheikh-Jabbari, "A Note on Noncommutative Chern-Simons Theories", hep-th/0102092; Dongsu Bak, Sung Ku Kim, Kwang-Sup Soh and Jae Hyung Yee, "Noncommutative Chern-Simons Solitons", hep-th/0102137;

[11] V.P. Nair and A.P. Polychronakos, "On Level Quantization for the Noncommutative Chern-Simons Theory", hep-th/0102181; D. Bak, K. Lee and J.-H. Park, "ChernSimons Theories on the Noncommutative Plane", hep-th/0102188.

[12] C.-S. Chu, Nucl. Phys. B580 (2000) 352; N. Grandi and G.A. Silva, "Chern-Simons action in noncommutative space", hep-th/0010113.

[13] L. Susskind, "The quantum Hall fluid and non-commutative Chern-Simons theory", hep-th/0101029; A.P. Polychronakos, "Quantum Hall states as matrix Chern-Simons theory", hep-th/0103013; S. Hellerman and M. Van Raamsdonk, "Quantum Hall Physics Equals Noncommutative Field Theory", hep-th/0103179.

[14] A. Brandhuber, M. Langer, M. Schweda, O. Piguet and S.P. Sorella, Phys. Lett. B300 (1993) 92 . 
[15] C.P. Martin, Phys. Lett. B263 (1991) 69.

[16] A. Basetto, M. Dalbosco and R. Soldati, Phys. Rev. D36 (1987)3138.

[17] Jaume Gomis and T. Mehen, Nucl.Phys. B591 (2000) 265-276; O. Aharony, Jaume Gomis and T. Mehen, JHEP 0009 (2000) 023; L. Alvarez-Gaumé, J.L.F. Barbón and R. Zwicky, "Remarks on Time-Space Noncommutative Field Theories", hepth/0103069.

[18] S. Mandelstam, Nucl. Phys. B213 (1983) 149; G. Leibbrandt, Phys. Rev. D29 (1984) 1699.

[19] A. Bassetto, M. Dalbosco, I. Lazzizera and R. Soldati, Phys. Rev. D31 (1985) 2012; P. Gaigg, M. Kreuzer, M. Schweda and O. Piguet, J. Math. Phys. 28 (1987) 2781; P. Gaigg and M. Kreuzer, Phys. Lett. B205 (1988) 530; G. Leibbrandt, Nucl. Phys. B320 (1988) 405; I. Lazzizera, Phys. Lett. B210 (1988) 188; H. Hüffel, P.V. Landshoff and J.C. Taylor, Phys. Lett. B217 (1989) 147; P.V. Landshoff, Phys. Lett. B227 (1989) 427.

[20] A. Basetto, G. Nardelli and R. Soldati, "Yang-Mills theories in algebraicnon-covariant gauges", World Scientific 1991; G. Leibbrandt, "Noncovariant Gauges", World Scientific, 1994.

[21] G. Leibbrandt and C.P. Martin, Nucl. Phys. B377 (1992) 593.

[22] W.F. Chen and G. Leibbrandt, "Vector Supersymmetry and Finite Quantum Correction of Chern-Simons Theory in the Light-Cone Gauge", hep-th/0012173.

[23] E.F. Moreno and F.A. Schaposnik, JHEP 0003 (2000) 032; K. Furuta and T. Inami, Mod. Phys. Lett. A15 (2000) 997; E.F. Moreno and F.A. Schaposnik, Nucl. Phys. B596 (2001) 439-458; A.M. Ghezelbash and S. Parvizi, Nucl. Phys. B592 (2001) 408416; A.R. Lugo, "Correlation functions in the non-commutative Wess-Zumino-Witten model', hep-th/0012268 .

[24] A. Das and M.M. Sheikh-Jabbari, "Absence of Higher Order Corrections to Noncommutative Chern-Simons Theory Coupling", hep-th/0103139. 